\title{
Coloring graphs of various maximum degree from random lists
}

\author{
Carl Johan Casselgren* \\ Department of Mathematics, Linköping University, \\ SE-581 83 Linköping, Sweden
}

\begin{abstract}
Let $G=G(n)$ be a graph on $n$ vertices with maximum degree $\Delta=\Delta(n)$. Assign to each vertex $v$ of $G$ a list $L(v)$ of colors by choosing each list independently and uniformly at random from all $k$-subsets of a color set $\mathcal{C}$ of size $\sigma=\sigma(n)$. Such a list assignment is called a random $(k, \mathcal{C})$-list assignment. In this paper, we are interested in determining the asymptotic probability (as $n \rightarrow \infty$ ) of the existence of a proper coloring $\varphi$ of $G$, such that $\varphi(v) \in L(v)$ for every vertex $v$ of $G$, a so-called $L$-coloring. We give various lower bounds on $\sigma$, in terms of $n, k$ and $\Delta$, which ensures that with probability tending to 1 as $n \rightarrow \infty$ there is an $L$-coloring of $G$. In particular, we show, for all fixed $k$ and growing $n$, that if $\sigma(n)=\omega\left(n^{1 / k^{2}} \Delta^{1 / k}\right)$ and $\Delta=O\left(n^{\frac{k-1}{k\left(k^{3}+2 k^{2}-k+1\right)}}\right)$, then the probability that $G$ has an $L$-coloring tends to 1 as $n \rightarrow \infty$. If $k \geq 2$ and $\Delta=\Omega\left(n^{1 / 2}\right)$, then the same conclusion holds provided that $\sigma=\omega(\Delta)$. We also give related results for other bounds on $\Delta$, when $k$ is constant or a strictly increasing function of $n$.
\end{abstract}

Keywords: list coloring, random list, coloring from random lists

\section{Introduction}

Given a graph $G$, assign to each vertex $v$ of $G$ a set $L(v)$ of colors (positive integers). Such an assignment $L$ is called a list assignment for $G$ and the sets $L(v)$ are referred to as lists or color lists. If all lists have equal size $k$, then $L$ is called a $k$-list assignment. We then want to find a proper vertex coloring $\varphi$ of $G$, such that $\varphi(v) \in L(v)$ for all $v \in V(G)$. If such a coloring $\varphi$ exists then $G$ is $L$-colorable and $\varphi$ is called an $L$-coloring. Furthermore, $G$ is called $k$-choosable if it is $L$-colorable for every $k$-list assignment $L$.

This particular variant of vertex coloring is known as list coloring and was introduced by Vizing [11] and independently by Erdös et al. [8].

In this paper we continue the study of the problem of coloring graphs from random lists introduced by Krivelevich and Nachmias [9, 10]: Assign lists of colors to the vertices of a graph $G=G(n)$ with $n$ vertices by choosing for each vertex $v$ its list $L(v)$ independently and uniformly at

${ }^{*}$ E-mail address: carl.johan.casselgren@liu.se Part of the work done while the author was a postdoc at University of Southern Denmark and at Mittag-Leffler Institute. Research supported by postdoctoral grants from SVeFUM and Mittag-Leffler Institute. 
random from all $k$-subsets of a color set $\mathcal{C}=\{1,2, \ldots, \sigma\}$. Such a list assignment is called a random $(k, \mathcal{C})$-list assignment for $G$. Intuitively it should hold that the larger $\sigma$ is, the more spread are the colors chosen for the lists and thus the more likely it is that we can find a proper coloring of $G$ with colors from the lists. The question that we address in this paper is how large $\sigma=\sigma(n)$ should be in order to guarantee that with high probability 1 (as $n \rightarrow \infty)$ there is a proper coloring of the vertices of $G$ with colors from the random list assignment.

This problem was first studied by Krivelevich and Nachmias [9, 10, for the case of powers of cycles and the case of complete bipartite graphs with parts of equal size $n$. In the latter case they showed that for all fixed $k \geq 2$, the property of having a proper coloring from a random $(k, \mathcal{C})$-list assignment exhibits a sharp threshold, and that the location of that threshold is exactly $\sigma(n)=2 n$ for $k=2$. In [4], we generalized the second part of this result and showed that for a complete multipartite graph with $s$ parts (fixed $s \geq 3$ ) of equal size $n$, the property of having a proper coloring from a random $(2, \mathcal{C})$-list assignment, has a sharp threshold at $\sigma(n)=2(s-1) n$.

Let $C_{n}^{r}$ be the $r$ th power of a cycle with $n$ vertices. For powers of cycles, Krivelevich and Nachmias proved the following theorem establishing a coarse threshold for the property of being colorable from a random list assignment.

Theorem 1.1. [9] Assume $r, k$ are fixed integers satisfying $r \geq k$ and let $L$ be a random $(k, \mathcal{C})$-list assignment for $C_{n}^{r}$. If we denote by $p_{C}(n)$ the probability that $C_{n}^{r}$ is L-colorable, then

$$
p_{C}(n)= \begin{cases}o(1), & \sigma(n)=o\left(n^{1 / k^{2}}\right), \\ 1-o(1), & \sigma(n)=\omega\left(n^{1 / k^{2}}\right) .\end{cases}
$$

In [5, 6] we generalized Theorem 1.1

Theorem 1.2. [5, 6] Let $G=G(n)$ be a graph on $n$ vertices with maximum degree bounded by some absolute constant, $k$ a fixed positive integer, and $L$ a random $(k, \mathcal{C})$-list assignment for $G$. If $\sigma(n)=\omega\left(n^{1 / k^{2}}\right)$, then $\mathbf{w h p} G$ is L-colorable.

Note that Theorem 1.2 is best possible for graphs with bounded maximum degree. Further results on the problem of coloring graphs from random lists appears in [2, 7].

In this paper we consider random $(k, \mathcal{C})$-list assignments for graphs $G=G(n)$ on $n$ vertices whose maximum degree $\Delta=\Delta(n)$ is an increasing function of $n$. We would like to suggest the following:

Conjecture 1.3. Let $G=G(n)$ be a graph on $n$ vertices with maximum degree at most $\Delta=\Delta(n)$, $k \geq 2$ a fixed positive integer, and $L$ a random $(k, \mathcal{C})$-list assignment for $G$.

(i) If $\Delta=o\left(n^{\frac{1}{k^{2}-k}}\right)$ and $\sigma=\omega\left(n^{1 / k^{2}} \Delta^{1 / k}\right)$, then whp $G$ is L-colorable.

(ii) If $\Delta=\Omega\left(n^{\frac{1}{k^{2}-k}}\right)$ and $\sigma=\omega(\Delta)$, then whp $G$ is L-colorable.

Note that when $\Delta$ is bounded then part (i) of the conjecture reduces to Theorem 1.2, Moreover, for the case $k=1$, it is easily seen that for a graph $G=G(n)$ on $n$ vertices with strictly increasing maximum degree $\Delta=\Delta(n)$, the coarse threshold for colorability from a random $(1, \mathcal{C})$-list assignment trivially occurs at $\sigma=n \Delta$.

We prove that part (i) of Conjecture 1.3 is true for a slightly more restrictive bound on $\Delta$ :

\footnotetext{
${ }^{1}$ An event $A_{n}$ occurs with high probability (abbreviated whp) if $\lim _{n \rightarrow \infty} \mathbb{P}\left[A_{n}\right]=1$.
} 
Theorem 1.4. Let $G=G(n)$ be a graph on $n$ vertices with maximum degree at most $\Delta=\Delta(n), k$ a fixed positive integer, and $L$ a random $(k, \mathcal{C})$-list assignment for $G$. If $\sigma(n)=\omega\left(n^{1 / k^{2}} \Delta^{1 / k}\right)$ and $\Delta=O\left(n^{\frac{k-1}{k\left(k^{3}+2 k^{2}-k+1\right)}}\right)$, then whp $G$ is L-colorable.

For the case $k=2$ we prove that both part (i) and (ii) of Conjecture 1.3 is true:

Theorem 1.5. Let $G=G(n)$ be a graph on $n$ vertices with maximum degree at most $\Delta=\Delta(n)$, and $L$ a random $(2, \mathcal{C})$-list assignment for $G$. If

(i) $\Delta=o\left(n^{1 / 2}\right)$ and $\sigma=\omega\left(n^{1 / 4} \Delta^{1 / 2}\right)$, or

(ii) $\Delta=\Omega\left(n^{1 / 2}\right)$ and $\sigma=\omega(\Delta)$,

then whp $G$ is L-colorable.

For a complete graph $K_{n}$ on $n$ vertices, the property of being colorable from a random $(2, \mathcal{C})$-list assignment has a sharp threshold at $\sigma(n)=2 n$ [5], and for $k \geq 3, K_{n}$ is whp colorable from a random $(k, \mathcal{C})$-list assignment if $\sigma \geq 1.223 n$ [6]. Thus Theorem 1.5 yields a better bound on $\sigma(n)$ for graphs $G$ with maximum degree $o(n)$.

In Section 2 we shall prove Theorem 1.4 and also give an example which shows that part (i) of Conjecture 1.3 (and therefore also Theorem 1.4) is sharp in the "coarse threshold sense": for each integer $n$ and each integer-valued function $\Delta=\Delta(n)$ satisfying $\Delta=O\left(n^{\frac{1}{k^{2}-k}}\right)$, there is a graph $H=H(n)$ with maximum degree $\Delta$ such that if $\sigma(n)=o\left(n^{1 / k^{2}} \Delta^{1 / k}\right)$ and $L$ is a random $(k, \mathcal{C})$-list assignment for $H$, then whp $H$ is not $L$-colorable. In Section 2 we also prove some related results for graphs with other bounds on the maximum degree.

Section 3 contains the proof of Theorem 1.5. Note also that part (ii) of the conjecture (and thus Theorem 1.5) is best possible in the same sense as Theorem 1.4, since a clique on $\Delta$ vertices requires at least $\Delta$ colors for a proper coloring.

In Section 4 we prove some related results for graphs with fixed girth greater than 3 , and we also prove a result for graphs with girth $\Omega(\log \log n)$ : for each constant $C>0$, there are constants $k_{0}=k_{0}(C)$ and $B=B(C)$, such that if $G$ is a graph with $n$ vertices, maximum degree at most $\Delta$, and girth $g \geq C \log \log n, k \geq k_{0}$ and $\sigma(n)>B \Delta$, then whp $G$ is colorable from a random $(k, \mathcal{C})$-list assignment. (For instance when $C=1$, then $k_{0}=9$ and $B=81 e^{7 / 2}$.)

In Section 5 we consider random list assignments where the uniform list size $k$ is a strictly increasing function of $n$. In particular, we present an analogue of Theorem 1.4 for lists of nonconstant size, and we prove that for any constant $C>0$, there is a constant $A=A(C)$, such that if $k \geq C \log n$ and $\sigma \geq A \Delta \log n$, then $G$ is whp colorable from a random $(k, \mathcal{C})$-list assignment.

\section{Proof of Theorem 1.4}

In this section we prove Theorem 1.4 and some related results. Our basic method in this paper is rather similar to the proof of the main result of [6], but we need to refine the method introduced in that paper, and use sharper estimates in many counting arguments.

Let $H$ be a graph and $L$ a list assignment for $H$. If $H$ is not $L$-colorable, but removing any vertex from $H$ yields an $L$-colorable graph, then $H$ is $L$-vertex-critical (or just $L$-critical). Obviously, if $L$ is a list assignment for a graph $G$, and $G$ is not $L$-colorable, then $G$ contains a connected induced $L$-critical subgraph. 
Suppose now that $H-w_{1}$ is $L$-colorable, where $w_{1}$ is some vertex of $H$. Given an $L$-coloring $\varphi$ of $H-w_{1}$, a path $P=w_{1} w_{2} \ldots w_{t}$ in $H$ is called $(\varphi, L)$-alternating if there are colors $c_{2}, c_{3}, \ldots, c_{t}$ such that $\varphi\left(w_{i}\right)=c_{i}$ and $c_{i} \in L\left(w_{i-1}\right), i=2, \ldots, t$. We allow such a path to have length 0 and thus only consist of $w_{1}$. The set of vertices which are adjacent to a vertex $x$ in a graph $G$ is denoted by $N_{G}(x)$. The following lemma was proved in [6].

Lemma 2.1. Let $F$ be a graph and $L$ a list assignment for $F$. If $F$ is $L$-critical, then for any vertex $v_{1} \in V(F), F-v_{1}$ has an L-coloring $\varphi$ that satisfies the following conditions:

(i) all vertices in $F$ lie on $(\varphi, L)$-alternating paths with origin at $v_{1}$;

(ii) for each color $c \in L\left(v_{1}\right)$, there is a vertex $w \in N_{F}\left(v_{1}\right)$, such that $\varphi(w)=c$.

(iii) Define a rank function $R: V(F) \rightarrow\{0,1, \ldots,|V(F)|-1\}$ on the vertices of $F$ by setting $R(u)=j$ if a shortest $(\varphi, L)$-alternating path from $v_{1}$ to $u$ has length $j$. Then for every vertex $x$ of $F-v_{1}$ and every color $c \in L(x) \backslash\{\varphi(x)\}$, there is either

(a) a vertex $y \in N_{F}(x)$ colored $\varphi(y)=c$ or

(b) a vertex $z \in N_{F}(x)$ such that $c \in L(z)$ and $R(z)<R(x)$.

For a rank function $R$ defined as in part (iii) of Lemma 2.1, we say that $R$ is the rank function on $V(F)$ induced by $L$ and $\varphi$.

Let $F$ be a connected induced subgraph of a graph $G, v_{1}$ a fixed vertex of $F$ and $R: V(F) \rightarrow$ $\{0,1, \ldots,|V(F)|-1\}$ a rank function on the vertices of $F$. The triple $\left(F, v_{1}, R\right)$ is proper, if $R\left(v_{1}\right)=0$ and $R(u)>0$ for each vertex $u \in V(F) \backslash\left\{v_{1}\right\}$, and if $R(u)=s$, then there is a vertex $x \in N_{F}(u)$ such that $R(x)=s-1$. (This definition of proper triple is slightly different from the one used in [6].) We also say that $\left(F, v_{1}, R\right)$ is a proper triple of $G$. Note that if $F, v_{1}$ and $R$ satisfies the conditions of Lemma 2.1 for some choice of $L$ and $\varphi$, then $\left(F, v_{1}, R\right)$ is proper. The next lemma gives an upper bound on the number of proper triples in a graph.

Lemma 2.2. Let $G$ be a graph on $n$ vertices whose maximum degree is at most $\Delta$. The number of proper triples $\left(F, v_{1}, R\right)$, such that $F$ is a subgraph of $G$ with $m$ vertices does not exceed

$$
n \Delta^{m-1}(m-1) !
$$

Proof. If $\left(F, v_{1}, R\right)$ is a proper triple, then by removing some edges of $F$ we can construct a tree $T$ with root $v_{1}$, such that if $u \in V(F)$ has rank $R(u)=r(1 \leq r \leq m-1)$, then $u$ is adjacent to a vertex $x$ in $T$ with $\operatorname{rank} R(x)=r-1$, and $v_{1}$ is the unique vertex of rank $R\left(v_{1}\right)=0$.

Moreover, given such a tree $T$ in $G$ with root $v_{1}$ and with a rank function $R: V(T) \rightarrow$ $\{0,1, \ldots, m-1\}$ satisfying these conditions, there is a uniquely determined proper triple $\left(F, v_{1}, R\right)$ with $V(F)=V(T)$, because $F$ is an induced subgraph of $G$. Hence, the number of proper triples of $G$ is bounded by the number of such trees in $G$ together with a rank function $R$. This latter quantity is bounded by

$$
n \Delta(2 \Delta)(3 \Delta) \ldots((m-1) \Delta)=n \Delta^{m-1}(m-1) !
$$

because there are $n$ ways of selecting $v_{1}$, then we have $\Delta$ choices for a neighbor of $v_{1}$ as the next vertex $v_{2}$ of $T$; thereafter, there are at most $(2 \Delta)$ ways of choosing an edge incident with $v_{1}$ or $v_{2}$ that connects one of these vertices with the next vertex of $T$, etc. 
Given a proper triple $\left(F, v_{1}, R\right)$ of a graph $G$ and a list assignment $L$ for $G$ such that $F$ is not $L$-colorable, we say that $\left(F, v_{1}, R\right)$ is $L$-bad (or just bad) if there is an $L$-coloring $\varphi$ of $F-v_{1}$, such that $F, v_{1}, R, L$ and $\varphi$ satisfy conditions (i)-(iii) of Lemma 2.1. In particular, $R$ is the rank function on $V(F)$ induced by $L$ and $\varphi$.

Consider a random $(k, \mathcal{C})$-list assignment for a graph $G$, where $\mathcal{C}=\{1,2, \ldots, \sigma\}$. The following lemma was proved in [6].

Lemma 2.3. Let $L$ be a random $(k, \mathcal{C})$-list assignment for a graph $G$ with maximum degree at most $\Delta$. If $\left(F, v_{1}, R\right)$ is a proper triple of $G$ with $m=|V(F)|$, then

$$
\mathbb{P}\left[\left(F, v_{1}, R\right) \text { is } L \text {-bad }\right] \leq \frac{\sigma^{m-1}\left(\begin{array}{c}
\Delta \\
k
\end{array}\right)\left(\begin{array}{c}
\Delta k \\
k-1
\end{array}\right)^{m-1}}{\left(\begin{array}{c}
\sigma \\
k
\end{array}\right)^{m}} .
$$

We are now in position to prove Theorem 1.4 .

Proof of Theorem 1.4. Let $G=G(n)$ be a graph on $n$ vertices with maximum degree at most $\Delta$ and let $L$ be a random $(k, \mathcal{C})$-list assignment for $G$, where $k$ is a fixed positive integer, and suppose further that $\Delta=O\left(n^{\frac{k-1}{k\left(k^{3}+2 k^{2}-k+1\right)}}\right)$ and $\sigma(n)=\omega\left(n^{1 / k^{2}} \Delta^{1 / k}\right)$. As pointed out above, the theorem is trivially true in the case when $k=1$. So in the following we shall assume that $k \geq 2$. We will show that whp $G$ has no connected induced $L$-critical subgraph. This suffices for proving the theorem. By Lemma 2.1, this means that we need to to prove that if $\sigma(n)=\omega\left(n^{1 / k^{2}} \Delta^{1 / k}\right)$, then whp $G$ does not contain an $L$-bad proper triple $\left(F, v_{1}, R\right)$. We will use first moment calculations.

If $\left(F, v_{1}, R\right)$ is $L$-bad, then $F$ has at least $k+1$ vertices. We first consider the case when $F$ has exactly $k+1$ vertices. It is not hard to see that if $F$ is not $L$-colorable and $|V(F)|=k+1$, then $F$ is a $(k+1)$-clique where all vertices have identical lists. The number of $(k+1)$-cliques in $G$ is at most $n \Delta^{k}$. Thus the expected number of $(k+1)$-cliques where the vertices get identical lists is at most

$$
n \Delta^{k}\left(\begin{array}{l}
\sigma \\
k
\end{array}\right)^{-k}
$$

which tends to 0 as $n \rightarrow \infty$, because $\sigma(n)=\omega\left(n^{1 / k^{2}} \Delta^{1 / k}\right)$. Hence, whp there is no bad proper triple $\left(F, v_{1}, R\right)$ in $G$ satisfying that $|V(F)|=k+1$.

Let us now consider the case when $|V(F)| \geq k+2$. First we show that if $\left(F, v_{1}, R\right)$ is bad, then whp $F$ contains at most $\Delta^{k^{2}+k}$ vertices. Consider a path $P$ on $r$ vertices in $G$ with origin at some vertex $v$. The probability that there is an $L$-coloring $\varphi$ of $P-v$, such that $P$ is $(\varphi, L)$-alternating is at most

$$
\frac{\sigma(\sigma-1)^{r-2}\left(\begin{array}{l}
\sigma-1 \\
k-1
\end{array}\right)^{2}\left(\begin{array}{l}
\sigma-2 \\
k-2
\end{array}\right)^{r-2}}{\left(\begin{array}{l}
\sigma \\
k
\end{array}\right)^{r}} \leq \frac{k^{2 r}}{\sigma^{r-1}},
$$

because there are at most $\sigma(\sigma-1)^{r-2}$ ways of choosing the proper coloring $\varphi$ and thereafter at most $\left(\begin{array}{c}\sigma-1 \\ k-1\end{array}\right)^{2}\left(\begin{array}{c}\sigma-2 \\ k-2\end{array}\right)^{r-2}$ ways of choosing the list assignment $L$ for $P$ so that $\varphi$ is an $L$-coloring of $P-v$ and $P$ is $(\varphi, L)$-alternating. Moreover, the number of distinct paths in $G$ on $r$ vertices is at most $n \Delta^{r-1}$. Therefore, the expected number of paths $P$ in $G$ on at least $k^{2}+k+1$ vertices, for which there is an $L$-coloring $\varphi$ of $P-v$ such that $P$ is $(\varphi, L)$-alternating is at most

$$
\sum_{r=k^{2}+k+1}^{n} \frac{n \Delta^{r-1} k^{2 r}}{\sigma^{r-1}}=o(1)
$$


since $\Delta=O\left(n^{\frac{k-1}{k\left(k^{3}+2 k^{2}-k+1\right)}}\right)$ and $\sigma(n)=\omega\left(n^{1 / k^{2}} \Delta^{1 / k}\right)$. Hence, by Markov's inequality, whp there is no $L$-coloring $\varphi$ of a subgraph of $G$ such that $G$ contains a $(\varphi, L)$-alternating path of length $k^{2}+k+1$.

Now, by Lemma 2.1, if $F$ is a subgraph of $G$ that belongs to a bad proper triple $\left(F, v_{1}, R\right)$, then there is an $L$-coloring $\varphi$ of $F-v_{1}$ such that all vertices of $F$ lie on $(\varphi, L)$-alternating paths with origin at $v_{1}$. Since whp the maximum length of such a path in $G$ is at most $k^{2}+k$, the maximum number of vertices in a subgraph of $G$ that is in a bad proper triple is whp at most

$$
1+\Delta+\Delta^{2}+\cdots+\Delta^{k^{2}+k-1} \leq \Delta^{k^{2}+k} .
$$

Let $X_{m}$ be a random variable counting the number of bad proper triples $\left(F, v_{1}, R\right)$ in $G$ such that $F$ has $m$ vertices and set

$$
X=\sum_{m=k+2}^{\Delta^{k^{2}+k}} X_{m} .
$$

Lemma 2.3 gives an upper bound on the probability that a given proper triple of $G$ on $m$ vertices is bad. Additionally, by Lemma 2.2 ,

$$
f(m) \leq n \Delta^{m-1}(m-1) !
$$

where $f(m)$ is the number of proper triples $\left(F, v_{1}, R\right)$ in $G$ such that $F$ has $m$ vertices. Let $p_{m}$ be the least number such that

$$
\mathbb{P}\left[\left(F, v_{1}, R\right) \text { is } L \text {-bad }\right] \leq p_{m},
$$

whenever $\left(F, v_{1}, R\right)$ is a proper triple in $G$ and $F$ has $m$ vertices. Since such a subgraph $F$ whp has at most $\Delta^{k^{2}+k}$ vertices if $\left(F, v_{1}, R\right)$ is $L$-bad, we conclude from Lemmas 2.2 and 2.3 that

$$
\begin{aligned}
\mathbb{P}[G \text { contains an } L \text {-bad proper triple }] & \leq \mathbb{E}[X]+o(1) \\
& \leq \sum_{m=k+2}^{\Delta^{k^{2}+k}} f(m) p_{m}+o(1) \\
& \leq \sum_{m=k+2}^{\Delta^{k^{2}+k}} n \Delta^{2 m-2}(m-1) ! \frac{\sigma^{m-1}\left(\begin{array}{l}
\Delta \\
k
\end{array}\right)\left(\begin{array}{l}
\Delta k \\
k-1
\end{array}\right)}{\left(\begin{array}{l}
\sigma \\
k
\end{array}\right)^{m}}+o(1) \\
& =O\left(\frac{n}{\sigma \Delta}\right) \sum_{m=k+2}^{\Delta^{k^{2}+k}}\left(\frac{m k^{k} \Delta^{k}}{\sigma^{k-1}}\right)^{m} \\
& =O\left(\frac{n \Delta^{k(k+2)}}{\sigma^{(k-1)(k+2)+1}}\right) \sum_{m=0}^{\infty}\left(\frac{k^{k} \Delta^{k^{2}+2 k}}{\sigma^{k-1}}\right)^{m} \\
& =o(1),
\end{aligned}
$$

provided that $\sigma(n)=\omega\left(n^{1 / k^{2}} \Delta^{1 / k}\right), \Delta=O\left(n^{\frac{k-1}{k\left(k^{3}+2 k^{2}-k+1\right)}}\right)$ and $k \geq 2$.

We now show that the bound on $\sigma$ in part (i) of Conjecture 1.3 (and also Theorem 1.4) is best possible in the "coarse threshold sense". We will show that for positive integers $k \geq 2$ and $n \geq k+1$ (large enough), and each non-constant increasing integer-valued function $\Delta=O\left(n^{\frac{1}{k^{2}-k}}\right)$, there is a 
graph $H=H(n, \Delta)$ with $n$ vertices and maximum degree $\Delta$ such that if $\sigma(n)=o\left(n^{1 / k^{2}} \Delta^{1 / k}\right)$ and $L$ is a random $(k, \mathcal{C})$-list assignment for $H$, then whp $H$ is not $L$-colorable.

So fix $k$, and let $n$ be a positive integer satisfying $n \geq k+1$ and assume that $\Delta$ satisfies $\Delta=O\left(n^{\frac{1}{k^{2}-k}}\right)$. We set $n_{\Delta}=\left\lfloor\frac{n}{\Delta+1}\right\rfloor$, and let $H$ be a graph on $n$ vertices which is the disjoint union of $n_{\Delta}$ complete graphs, each of which has $\Delta+1$ vertices, and possibly some isolated vertices. Let $J_{1}, \ldots, J_{n_{\Delta}}$ be the non-trivial components of $H$ and let $L$ be a random $(k, \mathcal{C})$-list assignment for $H$. We will prove that whp there is at least one $(k+1)$-clique in $H$ where all vertices have identical lists, which means that whp $H$ is not $L$-colorable. Note that we may assume that $\Delta \leq \sigma$, since otherwise, trivially there is no $L$-coloring of $H$.

Let $X$ be a random variable counting the number of $(k+1)$-cliques in $H$ where all vertices have identical lists. Then

$$
\mathbb{E}[X]=\left\lfloor\frac{n}{\Delta+1}\right\rfloor\left(\begin{array}{l}
\Delta+1 \\
k+1
\end{array}\right)\left(\begin{array}{l}
\sigma \\
k
\end{array}\right)^{-k}=\Theta\left(n(\Delta+1)^{k} \sigma^{-k^{2}}\right) .
$$

To prove that $\mathbb{P}[X>0]=1-o(1)$ we use the second moment method with the inequality due to Chebyshev in the following form:

$$
\mathbb{P}[Y=0] \leq \frac{\operatorname{Var}[Y]}{\mathbb{E}[Y]^{2}}
$$

valid for all non-negative random variables $Y$. Since $X$ is a sum of indicator random variables, we can use the following approach from [1].

Let $X=X_{1}+\cdots+X_{d}$, where each $X_{i}$ is the indicator random variable for the event that the vertices of a $(k+1)$-clique gets identical lists. Let $A_{i}$ be the event corresponding to $X_{i}$, that is, $X_{i}=1$ if $A_{i}$ occurs and $X_{i}=0$ otherwise. For indices $i, j$ we write $i \sim j$ if $i \neq j$ and the events $A_{i}, A_{j}$ are not independent. Set

$$
\Pi=\sum_{i \sim j} \mathbb{P}\left[A_{i} \wedge A_{j}\right]
$$

When $i \sim j$, we have

$$
\operatorname{Cov}\left[X_{i}, X_{j}\right]=\mathbb{E}\left[X_{i} X_{j}\right]-\mathbb{E}\left[X_{i}\right] \mathbb{E}\left[X_{j}\right] \leq \mathbb{E}\left[X_{i} X_{j}\right]=\mathbb{P}\left[A_{i} \wedge A_{j}\right]
$$

and when $i \neq j$ and not $i \sim j$ then $\operatorname{Cov}\left[X_{i}, X_{j}\right]=0$. Thus

$$
\operatorname{Var}[X] \leq \mathbb{E}[X]+\Pi
$$

and the following proposition follows from (4).

Claim 2.4. If $\mathbb{E}[X] \rightarrow \infty$ and $\Pi=o\left(\mathbb{E}[X]^{2}\right)$, then $\mathbb{P}[X>0]=1-o(1)$.

It is clear from (3) that $\mathbb{E}[X] \rightarrow \infty$ if $\sigma(n)=o\left(n^{1 / k^{2}} \Delta^{1 / k}\right)$. We now show that the second criterion of Claim 2.4 is satisfied. If $i \sim j$, then clearly $A_{i}$ and $A_{j}$ are events for cliques which are in the same component of $H$, and all vertices in these cliques have identical lists. Since $H$ has $n_{\Delta}$ components and two distinct $(k+1)$-cliques have at most $k$ vertices in common we have

$$
\Pi=O\left(n_{\Delta}\right) \sum_{l=1}^{k}\left(\begin{array}{c}
\Delta+1 \\
k+1+l
\end{array}\right)\left(\begin{array}{l}
\sigma \\
k
\end{array}\right)^{-(k+l)}=O\left(n(\Delta+1)^{k+2} \sigma^{-k(k+1)}\right),
$$


and thus $\Pi=o\left(\mathbb{E}[X]^{2}\right)$ as required. We conclude that Theorem 1.4 is best possible in the "coarse threshold sense".

Next, we will prove the following two propositions which show that weaker versions of Conjecture 1.3 hold for larger $\Delta$. As usual, $G=G(n)$ is a graph on $n$ vertices with maximum degree at most $\Delta=\Delta(n)$, and $L$ is a random $(k, \mathcal{C})$-list assignment for $G$, where $k \geq 2$ is a fixed positive integer.

Proposition 2.5. Suppose that $\alpha$ and $s$ are constants satisfying $1 \leq \alpha \leq 3$ and and $s \geq 2+\frac{2}{k-1}$, $\Delta=O\left(n^{1 / k^{\alpha}}\right)$, and $\sigma(n)=\omega\left(n^{1 / k^{\frac{\alpha+1}{2}}} \Delta^{s}\right)$. Then whp $G$ is L-colorable.

Proposition 2.6. If $\Delta=\Omega\left(n^{1 / k}\right)$ and $\sigma(n)=\omega\left(n^{\frac{1}{k}} \Delta\right)$, then whp $G$ is L-colorable.

We first prove Proposition 2.5. The proof is similar to the proof of Theorem 1.4 and therefore the proof will not be given in full detail.

Proof of Proposition 2.5 (sketch). Let $G=G(n)$ be a graph on $n$ vertices with maximum degree at most $\Delta=O\left(n^{1 / k^{\alpha}}\right)$, where $\alpha$ is a constant satisfying $1 \leq \alpha \leq 3$, and $k \geq 2$ is a fixed positive integer. Assume further that $s$ is a constant satisfying $s \geq 2+\frac{2}{k-1}$, and $\sigma=\omega\left(n^{1 / k^{\frac{\alpha+1}{2}}} \Delta^{s}\right)$. Note that the condition on $s$ implies that

$$
s k \geq 2 k+s .
$$

As in the proof of Theorem 1.4, we shall prove that $G$ whp has no bad proper triple.

Proceeding as in the proof of Theorem 1.4, one may first deduce that any $(\varphi, L)$-alternating path in $G \mathbf{w h p}$ has at most $k^{(\alpha+1) / 2}$ vertices, and thus if $(F, v, R)$ is a bad proper triple in $G$ then whp $F$ has at most $\Delta^{k^{(\alpha+1) / 2}}$ vertices. Using Lemmas 2.2 and 2.3 it now follows, as in the proof of Theorem 1.4, that the probability that $G$ has a bad proper triple tends to zero if the sum

$$
O\left(\frac{n}{\sigma \Delta}\right) \sum_{m=k+1}^{\Delta^{k^{\frac{\alpha+1}{2}}}}\left(\frac{m k^{k} \Delta^{k}}{\sigma^{k-1}}\right)^{m}
$$

tends to 0 as $n \rightarrow \infty$. Rewriting this sum yields that it is at most

$$
O\left(\frac{n \Delta^{k(k+1)}}{\sigma^{k^{2} \Delta}}\right) \sum_{m=0}^{\infty}\left(\frac{\Delta^{k^{\frac{\alpha+1}{2}}} k^{k} \Delta^{k} \sigma}{\sigma^{k}}\right)^{m}
$$

Setting $\sigma=n^{1 / k^{\frac{\alpha+1}{2}}} \Delta^{s}$, we have that the ratio of the geometric sum in (6) is at most

$$
\frac{\Delta^{k} \Delta^{s}}{\Delta^{(s-1) k}} \frac{\Delta^{k^{\frac{\alpha+1}{2}}} k^{k} n^{1 / k^{\frac{\alpha+1}{2}}}}{n^{1 / k^{\frac{\alpha-1}{2}}} \Delta^{k}} .
$$

The first factor in this expression is bounded by (5). As regards the second factor, this quantity is maximum when $\Delta=\Theta\left(n^{1 / k^{\alpha}}\right)$, and using the fact that $\alpha-1 \leq \alpha / 2+1 / 2$, when $\alpha \leq 3$, it follows that this factor is bounded as well.

It follows that if $\sigma=\omega\left(n^{1 / k^{\frac{\alpha+1}{2}}} \Delta^{s}\right)$, then the ratio of the geometric sum in (6) tends to zero as $n \rightarrow \infty$, and using this fact it is straightforward to verify that the expression (6) tends to zero. 
Let us now prove Proposition 2.6.

Proof of Proposition [2.6 (sketch). Let $k \geq 2$ be a positive integer and $G=G(n)$ be a graph on $n$ vertices with maximum degree at most $\Delta=\Omega\left(n^{1 / k}\right)$. We have to prove that if $\sigma(n)=\omega\left(n^{1 / k} \Delta\right)$ and $L$ is a random $(k, \mathcal{C})$-list assignment for $G$, then whp $G$ is $L$-colorable.

We will show that whp $G$ contains no connected induced $L$-critical subgraph. By Lemma 2.1 it suffices to prove that whp $G$ contains no vertex $v$ such that there are $k$ neighbors $u_{1}, \ldots, u_{k}$ of $v$ such that $c_{i} \in L\left(u_{i}\right)$, for $i=1, \ldots, k$, where $L(v)=\left\{c_{1}, \ldots, c_{k}\right\}$. The expected number of such vertices $v$ in $G$ is at most

$$
n\left(\begin{array}{l}
\Delta \\
k
\end{array}\right) \frac{\left(\begin{array}{l}
\sigma \\
k
\end{array}\right) k !\left(\begin{array}{c}
\sigma-1 \\
k-1
\end{array}\right)^{k}}{\left(\begin{array}{l}
\sigma \\
k
\end{array}\right)^{k+1}}=O\left(\frac{n \Delta^{k}}{\sigma^{k}}\right),
$$

which tends to 0 as $n \rightarrow \infty$, so the desired result follows from Markov's inequality.

\section{Lists of size 2}

In this section we prove Theorem 1.5. The proof is not very different from the proofs in the preceding section, but we use a somewhat different technique employed in [5] for proving results on random $(2, \mathcal{C})$-list assignments. Below we introduce some terminology and auxiliary results from that paper.

Let $P=v_{1} e_{1} v_{2} \ldots e_{d-1} v_{d}$ be a path. Then the sequence $C=v_{1} e_{1} v_{2} \ldots v_{d} e_{d} v_{1}$ is an ordered cycle if $e_{d}=v_{d} v_{1}$. Similarly, the sequence $D=v_{1} e_{1} v_{2} \ldots v_{d} e_{d} v_{j}$ is called an ordered lollipop if $e_{d}=v_{d} v_{j}$ and $j \in\{2, \ldots, d-2\}$. Note that an ordered cycle $C$ and an ordered lollipop $D$ is uniquely determined by a sequence of vertices (as is also a path). We may thus write $C=v_{1} \ldots v_{d} v_{1}$ for ordered cycles $C$, and similarly $D=u_{1} \ldots u_{j} \ldots u_{d} u_{j}$, for ordered lollipops $D$. Paths, ordered cycles and ordered lollipops will usually be referred to as sequences of vertices; however, sometimes we will refer to such sequences as graphs and then mean the graph consisting of the vertices and edges of the sequence. In particular, if $C$ is an ordered cycle or lollipop, then $V(C)$ and $E(C)$ are the sets of all vertices and edges in $C$, respectively.

Let $L$ be a 2-list assignment for a graph $G$ and let $C=v_{1} \ldots v_{d} v_{1}$ be an ordered cycle of $G$. Suppose that there are colors $c_{1}, \ldots, c_{d-1}$ such that $c_{1} \in L\left(v_{1}\right), L\left(v_{i}\right)=\left\{c_{i-1}, c_{i}\right\}, i=2, \ldots, d-1$ and $L\left(v_{d}\right)=\left\{c_{d-1}, c_{1}\right\}$. Then $C$ is L-alternating. Similarly, an ordered lollipop $D=u_{1} \ldots u_{j} \ldots u_{d} u_{j}$ in $G$ is $L$-alternating if there are colors $c_{1}, \ldots, c_{d-1}$ such that $c_{1} \in L\left(u_{1}\right)$ and $L\left(u_{i}\right)=\left\{c_{i-1}, c_{i}\right\}$, $i=2, \ldots, d-1$ and $L\left(u_{d}\right)=\left\{c_{d-1}, c_{j}\right\}$. For an $L$-alternating ordered cycle or ordered lollipop $D$, the common color $c_{1}$ of the lists of the first two vertices of $D$ will be referred to as the first color of $D$.

The following lemma was proved in [5].

Lemma 3.1. Let $G$ be a graph and $L$ a 2-list assignment for $G$. If $G$ is not $L$-colorable, then there are subgraphs $H_{1}$ and $H_{2}$ of $G$, such that for $i=1,2$ :

(i) $H_{i}$ is either an L-alternating ordered cycle or an L-alternating ordered lollipop;

(ii) there is a vertex $v$ of $G$ with $L(v)=\left\{c_{1}, c_{2}\right\}$, such that the first vertex of $H_{i}$ is $v$ and the first color is $c_{i}$;

(iii) the second vertex of $H_{1}$ and the second vertex of $H_{2}$ are distinct. 
Consider a graph $G$ with a 2-list assignment $L$. The pair $F=\left(H_{1}, H_{2}\right)$ is then called a proper pair if $H_{1}$ is an ordered cycle or ordered lollipop in $G, H_{2}$ is an ordered cycle or ordered lollipop in $G$ and $H_{1}$ and $H_{2}$ have a common first vertex. Moreover, a proper pair $F=\left(H_{1}, H_{2}\right)$ is $(L, 2)$-bad (or just 2-bad) if $H_{1}, H_{2}$ and $L$ satisfy the conditions (i)-(iii) of Lemma 3.1. Note that if $F=\left(H_{1}, H_{2}\right)$ is a 2 -bad proper pair, then $F$ is not $L$-colorable.

By slight abuse of terminology we will sometimes refer to proper pairs $F=\left(H_{1}, H_{2}\right)$ in $G$ as subgraphs of $G$. For such a proper pair $F=\left(H_{1}, H_{2}\right),\left|V\left(H_{1}\right) \cup V\left(H_{2}\right)\right|$ is the number of vertices of $F$.

Remark 3.2. If $F=\left(H_{1}, H_{2}\right)$ is a 2-bad proper pair with a common first vertex $v$, then trivially there is a subgraph $J$ of $F$ such that $J-v$ has an $L$-coloring $\varphi$. Moreover, if $R$ is the rank function induced by $L$ and $\varphi$ as in Section 2, then $(J, v, R)$ is a bad proper triple. So for a graph with a 2-list assignment $L$, Lemma 3.1 provides a stronger characterization than Lemma 2.1 of which 2-list assignments do not contain a proper coloring of the graph.

Suppose that $H_{1}$ is an ordered cycle or lollipop and $H_{2}$ is an ordered cycle or lollipop. Assume further that $H_{1}$ contains the vertices $v, v_{2}, \ldots, v_{d_{1}}$ and that the vertices lie in this order along $H_{1}$. Suppose that $v_{i} \in V\left(H_{1}\right) \cap V\left(H_{2}\right), v_{i} \neq v$ and let $u$ be the vertex that precedes $v_{i}$ along $H_{2}$. If $u v_{i} \notin E\left(H_{1}\right)$, then $v_{i}$ is called a non-consecutive common vertex of $H_{1}$ and $H_{2}$. Otherwise, if $u v_{i} \in H_{1}$ then $v_{i}$ is called a consecutive common vertex.

The following two lemmas are essentially variants of Lemmas 13 and 14 in [5], respectively; since we have not found a way to deduce them directly from those lemmas, we provide brief sketches of the proofs (for details see [5]).

Lemma 3.3. Let $G$ be a graph and let $L$ be a random $(2, \mathcal{C})$-list assignment for $G$. Suppose that $F=\left(H_{1}, H_{2}\right)$ is a proper pair in $G$ on $l$ vertices and $r$ non-consecutive common vertices. Then

$$
\mathbb{P}[F \text { is } 2 \text {-bad }] \leq \frac{2^{l+2 r}}{\sigma^{l-1}(\sigma-1)^{2+r}} .
$$

Proof (sketch). Let $l_{1}=\left|V\left(H_{1}\right)\right|$ and $l_{2}=\left|V\left(H_{2}\right) \backslash V\left(H_{1}\right)\right|$. We will prove the lemma assuming that $l_{2}>0$. If $l_{2}=0$, then a similar argument applies. Suppose that $H_{1}$ is an ordered cycle or an ordered lollipop on the vertices $v, v_{2}, v_{3}, \ldots, v_{l_{1}}$ and that the vertices lie in that order along $H_{1}$. Assume further that there are $r_{1}$ non-consecutive common vertices $u$ of $H_{1}$ and $H_{2}$ such that the predecessor of $u$ along $H_{2}$ is in $V\left(H_{1}\right)$. Denote the set of these vertices by $R_{1}$.

There are $\sigma(\sigma-1)$ ways of choosing the list $L(v)$ and selecting one of the colors of $L(v)$ as the first color of $H_{1}$. Then there are at most $(\sigma-1)^{l_{1}-r_{1}-2} 2^{2 r_{1}}$ ways of choosing the lists for $H_{1}-v$, so that $H_{1}$ is $L$-alternating, and so that the list of every vertex of $R_{1}$ has a color in common with the list of its predecessor along $H_{2}$; we choose the lists for the vertices $v_{2}, v_{3}, \ldots, v_{l_{1}}$ sequentially except that for any pair of vertices $v_{i}$ and $v_{j}(i<j)$ that are adjacent on $H_{2}$ and satisfying that one of $v_{i}$ and $v_{j}$ is a non-consecutive common vertex, we fix one color of $L\left(v_{j}\right)$ immediately after choosing the colors for $L\left(v_{i}\right)$.

So in total we have at most $\sigma(\sigma-1)(\sigma-1)^{l_{1}-r_{1}-2} 2^{2 r_{1}}$ choices for the lists of $H_{1}$.

Since there are $r-r_{1}$ non-consecutive common vertices of $H_{1}$ and $H_{2}$ that are not in $R_{1}$, there are thereafter at most $(\sigma-1)^{l_{2}-1-\left(r-r_{1}\right)} 2^{r-r_{1}}$ ways of choosing the lists of the vertices in $V\left(H_{2}\right) \backslash V\left(H_{1}\right)$ so that $H_{2}$ is $L$-alternating as well. Since, in total, there are $\left(\begin{array}{c}\sigma \\ 2\end{array}\right)^{l}$ ways of choosing the restriction of $L$ to the vertices in $F$, the result follows.

Lemma 3.4. Let $G$ be a graph on $n$ vertices with maximum degree $\Delta$. The number of proper pairs $F=\left(H_{1}, H_{2}\right)$ on $l$ vertices in $G$ with $r$ non-consecutive vertices is at most $n \Delta^{l-1+r} 2^{l}$. 
Proof (sketch). Let $\left|V\left(H_{1}\right)\right|=l_{1}$ and $\left|V\left(H_{2}\right) \backslash V\left(H_{1}\right)\right|=l_{2}$. The first vertex $v$ of $H_{1}$ and $H_{2}$ can be chosen in at most $n$ ways. After that, there are at most $\Delta^{l_{1}-1}$ choices for the rest of $H_{1}$. Thereafter, there are at most $2^{l_{1}} \Delta^{l_{2}+r}$ choices for the vertices of $H_{2}-v$, because there are at most $l_{1}$ vertices of $H_{1}$ which might be consecutive common vertices of $H_{1}$ and $H_{2}$, and $l_{2}+r$ other vertices of $H_{2}$.

Proof of Theorem 1.5. Let $G=G(n)$ be a graph on $n$ vertices with maximum degree at most $\Delta$ and let $L$ be a random $(2, \mathcal{C})$-list assignment for $G$. By Lemma 3.1, it suffices to prove that if either $\Delta=\Omega\left(n^{1 / 2}\right)$ and $\sigma=\omega(\Delta)$ or $\Delta=o\left(n^{1 / 2}\right)$ and $\sigma=\omega\left(n^{1 / 4} \Delta^{1 / 2}\right)$, then whp $G$ does not contain a 2 -bad proper pair. We will use easy first moment calculations.

Any 2-bad proper pair contains at least 3 vertices. For $l \in\{3, \ldots, n\}$ and $r \in\{1, \ldots, l\}$, let $X_{l, r}$ be a random variable counting the number of 2-bad proper pairs with $l$ vertices and $r$ nonconsecutive common vertices in $G$, and let

$$
X=\sum_{l=3}^{n} \sum_{r=0}^{l} X_{l, r} .
$$

By Lemma 3.4, $f(l, r) \leq n \Delta^{l-1+r} 2^{l}$, where $f(l, r)$ is the number of proper pairs on $l$ vertices and $r$ non-consecutive common vertices in $G$. Let $p_{l, r}$ be the least number such that $\mathbb{P}\left[F_{l, r}\right.$ is 2 -bad $] \leq p_{l, r}$, whenever $F_{l, r}$ is a proper pair on altogether $l$ vertices and $r$ non-consecutive common vertices in $G$. By Lemmas 3.3 and 3.4 we have

$$
\begin{aligned}
\mathbb{P}[G \text { contains a 2-bad proper pair }] \leq \mathbb{E}[X] & \leq \sum_{l=3}^{n} \sum_{r=0}^{l} f(l, r) p_{l, r} \\
& \leq \sum_{l=3}^{n} \sum_{r=0}^{l} n \Delta^{l-1+r} 2^{l} \frac{2^{l+2 r}}{\sigma^{l-1}(\sigma-1)^{2+r}} \\
& =O\left(\frac{n \Delta^{2}}{\sigma^{4}}\right) \sum_{l=0}^{n}\left(\frac{4 \Delta}{\sigma}\right)^{l} \\
& =o(1),
\end{aligned}
$$

provided that $\Delta=o\left(n^{1 / 2}\right)$ and $\sigma(n)=\omega\left(n^{1 / 4} \Delta^{1 / 2}\right)$, or $\Delta=\Omega\left(n^{1 / 2}\right)$ and $\sigma=\omega(\Delta)$.

Consider a random $(2, \mathcal{C})$-list assignment for a graph $G=G(n)$ on $n$ vertices with maximum degree at most $\Delta=\Delta(n)$ and girth $g$. A 2-bad proper pair in $G$ has at least $g$ vertices, and by proceeding precisely as in the proof of Theorem 1.5 we can prove the following result which yields a better bound than the one of Proposition 2.6 if $k \leq g$.

Proposition 3.5. Let $G=G(n)$ be a graph on $n$ vertices with maximum degree at most $\Delta=\Delta(n)$ and girth at least $g$, where $g$ is a fixed positive integer, and let $L$ be a random $(2, \mathcal{C})$-list assignment for $G$. If $\sigma(n)=\omega\left(n^{\frac{1}{g+1}} \Delta\right)$, then whp $G$ is L-colorable.

In the next section we shall prove several other results for graphs with girth greater than 3 .

\section{Graphs with girth greater than three}

For the graph $H$ in the example in Section 2 showing that Theorem 1.4 is best possible, the threshold for the property of being colorable from a random $(k, \mathcal{C})$-list assignment coincides with 
the threshold for disappearence of $(k+1)$-cliques where each vertex has the same list; that is, when $\sigma(n)=\omega\left(n^{1 / k^{2}} \Delta^{1 / k}\right)$, then whp $H$ has no such cliques, and when $\sigma(n)=o\left(n^{1 / k^{2}} \Delta^{1 / k}\right)$, then whp $H$ contains a $(k+1)$-clique where the vertices have identical lists.

As in [5, 6], for a graph $G=G(n)$ on $n$ vertices with girth $g \geq 4$ (and thus with no $(k+1)$ cliques if $k \geq 2$ ) it is possible to establish a better bound on $\sigma(n)$ than those given by Theorem 1.4. Propositions 2.5] and 2.6, which implies list colorability of $G$ from a random $(k, \mathcal{C})$-list assignment. Indeed, the coarse threshold $n^{1 / k^{2}} \Delta^{1 / k}$ in Theorem 1.4 is essentially due to the fact that the probability that a $(k+1)$-clique has a list assignment where all lists are equal is $\left(\begin{array}{l}\sigma \\ k\end{array}\right)^{-k}$ and the maximum number of $(k+1)$-cliques in a graph with maximum degree $\Delta$ is roughly $n \Delta^{k}$, if $\Delta$ is sufficiently small compared to $n$.

If $L$ is a $k$-list assignment for a graph $G$, then any $L$-critical subgraph of $G$ which is not a $(k+1)$-clique has strictly more than $k+1$ vertices, and employing this fact we can use the very same methods as in Section 2 and 3, to prove better bounds on $\sigma$ which ensures that whp $G$ is colorable from a random $(k, \mathcal{C})$-list assignment. Considering graphs with large girth is one way of increasing the minimum number of vertices in an $L$-critical graph - which is the most relevant parameter - and one can of course derive corresponding results for other families of graphs.

We emphasize that the results in this section are probably not best possible for any values of $\Delta$ or $k$. To prove sharp results, a first step would be to investigate how many vertices the smallest non- $k$-choosable graph with girth $g$ has, and then estimate the number of $k$-list assignments that do not contain a proper coloring of such a graph.

We will proceed as in the proof of Theorem 1.4 and use first moment calculations to show that if $L$ is a random $(k, \mathcal{C})$-list assignment for a graph $G=G(n)$ on $n$ vertices with girth $g$, where $k$ and $g$ are fixed integers satisfying $k \geq 3$ and $g>3$, respectively, $\Delta=\Delta(n)$ is sufficiently small, and $\sigma=\sigma(n)$ is large enough, then whp $G$ has no connected induced $L$-critical subgraph and thus is whp $L$-colorable. (The case $k=2$ was considered in the previous section.)

Now, any $L$-critical subgraph of $G$ has minimum degree $k+1$ (which follows from the listcoloring version of Brooks' theorem since $k \geq 3$ ), and as pointed out in [6] (see also [3]), such a graph contains at least $Q(k+1)$ vertices, where $Q(k)=Q(k, g)$ satisfies

$$
Q(k)= \begin{cases}1+k\left(1+(k-1)+(k-1)^{2}+\cdots+(k-1)^{\frac{g-3}{2}}\right), & \text { if } g \text { is odd } \\ 2\left(1+(k-1)+(k-1)^{2}+\cdots+(k-1)^{\frac{g-2}{2}}\right), & \text { if } g \text { is even }\end{cases}
$$

and thus

$$
Q(k)= \begin{cases}1+\frac{k}{k-2}\left((k-1)^{(g-1) / 2}-1\right) & \text { if } g \text { is odd } \\ \frac{2}{k-2}\left((k-1)^{g / 2}-1\right) & \text { if } g \text { is even. }\end{cases}
$$

Suppose that $\sigma=\omega\left(n^{\frac{1}{(k-1) Q(k+1)+1}} \Delta^{s}\right)$, where $s$ is a fixed integer satisfying $s \geq 1+\frac{1}{k-1}$ and $\Delta=O\left(n^{\frac{1}{k Q^{2}(k+1)}}\right)$. By proceeding as in the proof of Theorem 1.4, it is not hard to show that if $F$ is $L$-critical and thus by Lemma 2.1 belongs to an $L$-bad proper triple $(F, v, R)$ of $G$, then whp $F$ contains at most $\Delta^{(k-1) Q(k+1)+1}$ vertices. Hence, by Lemma 2.1, it suffices to prove that whp $G$ contains no bad proper triple $(F, v, R)$ such that

$$
Q(k+1) \leq|V(F)| \leq \Delta^{(k-1) Q(k+1)+1} .
$$

Set $h(k)=\Delta^{(k-1) Q(k+1)+1}$, and let $Z_{m}$ be a random variable counting the number of $L$-bad 
proper triples $(F, v, R)$ in $G$ such that $F$ has $m$ vertices, and set

$$
Z=\sum_{m=Q(k+1)}^{h(k)} Z_{m} .
$$

Using Lemmas 2.2 and 2.3 we may now conclude that

$\mathbb{P}[G$ contains an $L$-bad proper triple $] \leq \mathbb{E}[Z]+o(1)$

$$
\begin{aligned}
& \leq \sum_{m=Q(k+1)}^{h(k)} n \Delta^{m-1}(m-1) ! \frac{\sigma^{m-1}\left(\begin{array}{c}
\Delta \\
k
\end{array}\right)\left(\begin{array}{c}
\Delta k \\
k-1
\end{array}\right)^{m-1}}{\left(\begin{array}{c}
\sigma \\
k
\end{array}\right)^{m}}+o(1) \\
& =O\left(\frac{n}{\sigma \Delta}\right) \sum_{m=Q(k+1)}^{h(k)}\left(\frac{m k^{k} \Delta^{k}}{\sigma^{k-1}}\right)^{m} \\
& =O\left(\frac{n \Delta^{k Q(k+1)}}{\sigma^{(k-1) Q(k+1)+1}}\right) \sum_{m=0}^{\infty}\left(\frac{k^{k} \Delta^{(k-1) Q(k+1)+k+1}}{\sigma^{k-1}}\right)^{m} \\
& =o(1),
\end{aligned}
$$

provided that $\sigma(n)=\omega\left(n^{\frac{1}{(k-1) Q(k+1)+1}} \Delta^{s}\right), s \geq 1+1 /(k-1)$ and $\Delta=O\left(n^{\frac{1}{k Q^{2}(k+1)}}\right)$. Let us determine $(k-1) Q(k+1)+1$ explicitly for some small values of $g$. When $g=4$, then if

$$
\sigma(n)=\omega\left(n^{\frac{1}{2 k^{2}-1}} \Delta^{s}\right) \text { and } \Delta=O\left(n^{\frac{1}{4\left(k^{3}+2 k^{2}+k\right)}}\right)
$$

then whp there is an $L$-coloring of $G$, and when $g=5$, then it suffices to require that

$$
\sigma(n)=\omega\left(n^{1 /\left(k^{3}+k^{2}-1\right)} \Delta^{s}\right) \text { and } \Delta=O\left(n^{\frac{1}{k^{5}+4 k^{4}+8 k^{3}+8 k^{2}+4 k}}\right) .
$$

We collect these results in the following proposition.

Proposition 4.1. Let $G=G(n)$ be a graph on $n$ vertices with maximum degree $\Delta$ and girth at least $g$, where $g$ is a fixed positive integer. Suppose that $k$ is a fixed integer satisfying $k \geq 3$ and that $L$ is a random $(k, \mathcal{C})$-list assignment for $G$. Moreover, let $s$ be a fixed integer satisfying $s \geq 1+\frac{1}{k-1}$

(i) If $g=4, \sigma(n)=\omega\left(n^{\frac{1}{2 k^{2}-1}} \Delta^{s}\right)$, and $\Delta=O\left(n^{\frac{1}{4 k^{3}+8 k^{2}+4 k}}\right)$, then whp $G$ has an L-coloring.

(ii) If $g=5, \sigma(n)=\omega\left(n^{1 /\left(k^{3}+k^{2}-1\right)} \Delta^{s}\right)$, and $\Delta=O\left(n^{\frac{1}{k^{5}+4 k^{4}+8 k^{3}+8 k^{2}+4 k}}\right)$, then whp $G$ has an L-coloring.

(iii) If $g>5$, then there are polynomials $P(k)$ and $R(k)$ in $k$ of degree $\lceil g / 2\rceil$ and $2\lceil g / 2\rceil-1$, respectively, such that if $\sigma(n)=\omega\left(n^{1 / P(k)} \Delta^{s}\right)$, and $\Delta=O\left(n^{1 / R(k)}\right)$, then whp $G$ is $L$ colorable. Moreover,

$$
P(k)=(k-1) Q(k+1)+1 \text { and } R(k)=k Q^{2}(k+1),
$$

where $Q(k)$ is given by (7). 
For graphs with no restriction on the maximum degree, we shall prove the following, which yields a slightly weaker bound on $\sigma$.

Proposition 4.2. Let $G=G(n)$ be a graph on $n$ vertices with maximum degree $\Delta=\Delta(n)$ and girth at least $g$, where $g>3$ is a fixed positive integer. Suppose further that $k$ is a fixed integer

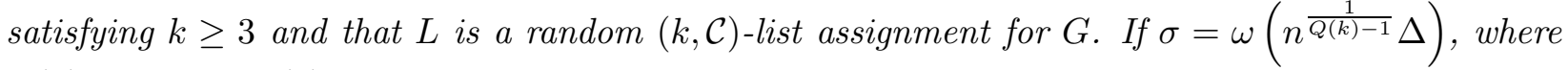
$Q(k)$ is given by (7), then whp $G$ is L-colorable.

For the proof of the above result we need some new tools. The distance between two vertices in a graph $G$ is the number of edges in a shortest path in $G$ between them.

Given a graph $G$ with girth $g$, where $g$ is a positive integer, an odd rooted k-proper tree in $G$ is a rooted tree $T$ with root $v$ such that $v$ has $k$ neighbors in $T$, and for each $i=1, \ldots,\lfloor(g-3) / 2\rfloor$, each vertex at distance $i$ from $v$ has exactly $k-1$ neighbors at distance $i+1$ from $v$, and no vertex of distance $\lfloor(g-1) / 2\rfloor$ from $v$ has a neighbor at greater distance from $v$. Note that the subgraph of $G$ induced by all vertices at distance at most $\lfloor(g-3) / 2\rfloor$ from $v$ in $T$ is a tree, and that no two vertices in $G$ at distance $\lfloor(g-3) / 2\rfloor$ from $v$ has a common neighbor at distance $\lfloor(g-1) / 2\rfloor$ from $v$, because $G$ has girth $g$.

For an even integer $g$, and a graph $G$ with girth $g$ we define the concept of an even $k$-proper tree as follows: let $u$ and $v$ be adjacent vertices and let $T_{u}$ and $T_{v}$ be two vertex-disjoint odd rooted $k$-proper trees in $G$ with roots $u$ and $v$, respectively, except for the fact that $u$ and $v$ has exactly $k-1$ neighbors in $T_{u}$ and $T_{v}$, respectively. An even rooted $k$-proper tree with root $v$ and semiroot $u$ is the union of $T_{u}$ and $T_{v}$ along with the edge $u v$.

It is straightforward to prove that for odd $g$, an odd $k$-proper rooted tree has exactly $Q(k)$ vertices, and that for even $g$, an even $k$-proper rooted tree has exactly $Q(k)$ vertices, where $Q(k)$ is given by (7). (Indeed, this formula can be proved by considering a breadth-first search tree with root at some vertex in a graph with minimum degree $k$.)

Suppose now that $L$ is a $k$-list assignment for $G$, and that $T$ is an odd rooted $k$-proper tree $T$ in $G$ with root $v$. Then $T$ is (tree, $L$ )-bad (or just tree-bad) if there is an $L$-coloring $\varphi$ of $T-v$ such that

(i) $L(v)=\left\{\varphi(x): x \in N_{T}(v)\right\}$

(ii) for every $i=1, \ldots,\lfloor(g-3) / 2\rfloor$, every vertex $x \in V(T)$ at distance $i$ from $v$ satisfies that

$$
L(x) \backslash\{\varphi(x)\}=\left\{\varphi(y): y \in N_{T}(x) \text { and the distance between } y \text { and } v \text { is } i+1\right\} .
$$

Suppose now that $g$ is even and that $T$ is an even rooted $k$-proper tree with root $v$ and semiroot $u$. Then $T$ is (tree, $L$ )-bad (or just tree-bad) if there is an $L$-coloring $\varphi$ of $T-v$ such that

(iii) $L(v)=\left\{\varphi(x): x \in N_{T}(v)\right\}$

(iv) for every $i=1, \ldots,(g-4) / 2$, every vertex $x \in V(T)$ at distance $i$ from $v$ satisfies that

$$
L(x) \backslash\{\varphi(x)\}=\left\{\varphi(y): y \in N_{T}(x) \text { and the distance between } y \text { and } v \text { is } i+1\right\},
$$

and for every $i=1, \ldots,(g-4) / 2$, every vertex $z \in V(T)$ at distance $i$ from $u$ satisfies that

$$
L(z) \backslash\{\varphi(z)\}=\left\{\varphi(w): w \in N_{T}(z) \text { and the distance between } w \text { and } u \text { is } i+1\right\} .
$$


Lemma 4.3. Let $G$ be a graph with girth $g$ and $L$ a $k$-list assignment for $G$, where $g$ and $k$ are positive integers. If $G$ is not $L$-colorable and $g$ is odd (even), then there is a tree-bad odd (even) rooted $k$-proper tree $T$ in $G$.

Proof. We shall prove the lemma in the case when $g$ is odd. The case when $g$ is even can be done similarly. If $G$ is not $L$-colorable, then it contains a vertex-minimal conncected induced $L$-critical subgraph $H$. Let $v$ be a vertex of $H$; then $H-v$ has an $L$-coloring $\theta$. For such an $L$-coloring $\psi$ of $H-v$, let $W^{\psi}$ be the set of vertices $u$ in $H$ for which there is an $(\psi, L)$-alternating path of length at most $\frac{g-1}{2}$ with origin at $v$ and terminus $u$. For a vertex $u$ in $H\left[W^{\theta}\right]$ we say that $u$ is at level $r$ if the distance between $u$ and $v$ is $r$. We shall prove by contradiction that $H\left[W^{\theta}\right]$ contains an tree-bad odd rooted $k$-proper tree $T$.

If $\psi$ is an $L$-coloring of $H-v$, then the subgraph of $H\left[W^{\psi}\right]$ induced by all vertices at level at most $(g-3) / 2$ in $H\left[W^{\psi}\right]$ is a tree and no two vertices at level $(g-3) / 2$ has a common neighbor at level $(g-1) / 2$, because $G$ has girth $g$.

Now consider the subgraph $H\left[W^{\theta}\right]$. For each color $c \in L(v)$, there is clearly a neighbor of $v$ that is colored $c$ under $\theta$ (since otherwise $H$ is $L$-colorable). Suppose that $H\left[W^{\theta}\right]$ does not contain a treebad odd rooted $k$-proper tree. We shall prove that this implies that $H$ is $L$-colorable, establishing the required contradiction. Let $\mathcal{T}$ be the set of all subgraphs $T_{\theta}$ of $H\left[W^{\theta}\right]$ such that

(a) $v \in V\left(T_{\theta}\right)$,

(b) either 0 or exactly $k$ neighbors of $v$ are in $T_{\theta}$, and in the latter case $L(v)=\left\{\theta(x): x \in N_{T_{\theta}}(v)\right\}$,

(c) for every vertex $u$ of $T_{\theta}$ at level $q$, where $1 \leq q \leq \frac{g-3}{2}, T_{\theta}$ contains exactly 0 or $k-1$ neighbors of $u$ at level $q+1$,

(d) for every vertex $u \in V\left(T_{\theta}\right)$ and every color $c \in L(u) \backslash\{\theta(u)\}$, if $u$ is at level $q$, where $1 \leq q \leq \frac{g-3}{2}$, and $u$ has $k-1$ neighbors in $T_{\theta}$ at level $q+1$, then there is a neighbor $x$ of $u$ at level $q+1$ with $\theta(x)=c$.

We choose an element $T_{\theta}$ from $\mathcal{T}$ such that the shortest maximal path with origin at $v$ in $T_{\theta}$ has maximum length. If $\mathcal{T}$ contains several elements with shortest maximal paths of equal length, then we choose $T_{\theta}$ to be an element of $\mathcal{T}$ with the minimum number of such paths. We say that such a subgraph of $H\left[W^{\theta}\right]$ is path-maximal.

If each maximal path in $T_{\theta}$ with origin at $v$ has length at least $\frac{g-1}{2}$, then $H\left[W^{\theta}\right]$ clearly contains a tree-bad odd rooted $k$-proper tree; so suppose that there is some maximal path in $T_{\theta}$ with origin at $v$ of length strictly less than $\frac{g-1}{2}$. Let $P=v w_{1} w_{2} \ldots w_{j}$ be such a shortest path in $T_{\theta}$ and suppose further that $\theta\left(w_{i}\right)=c_{i}, i=1, \ldots, j$.

Now, if $v=w_{j}$, then $H$ is $L$-colorable; a contradiction and the desired result follows. So suppose that $v \neq w_{j}$. Since $T_{\theta}$ is path-maximal, there is a color $c_{j+1} \in L\left(w_{j}\right) \backslash\left\{\theta\left(w_{j}\right)\right\}$ such that no vertex in $H\left[W^{\theta}\right]$ at level $j+1$ is adjacent to $w_{j}$ and colored $c_{j+1}$. We call such a color a free color of $w_{j}$. In fact, since $T_{\theta} \in \mathcal{T}$ and $T_{\theta}$ is path-maximal, there is some color $c \in L\left(w_{j-1}\right) \backslash\left\{\theta\left(w_{j-1}\right)\right\}$ such that each neighbor of $w_{j-1}$ at level $j$ in $H\left[W^{\theta}\right]$ colored $c$ has a free color.

Let $H^{\prime}$ be the subgraph of $H\left[W^{\theta}\right]$ consisting of all vertices $u$ for which there is a $(\theta, L)$-alternating path of length at most $j$ with origin at $v$, terminus at $u$ and whose second vertex is colored $c_{1}$. Note that $H^{\prime}$ is a tree and denote by $\left\{a_{1}, \ldots, a_{r}\right\}$ the set of neighbors of $v$ in $H^{\prime}$. For $i=1, \ldots, r$, denote by $H_{i}^{\prime}$ the subgraph of $H^{\prime}$ consisting of all vertices $u$ for which there is a $(\theta, L)$-alternating path of length at most $j$ with origin at $v$, terminus at $u$ and whose second vertex is $a_{i}$.

For $i=1, \ldots, r$, let $T_{i}$ be a path-maximal tree in $H_{i}^{\prime}$ satisfying (a) and (c)-(d) (with $T_{i}$ in place of $T_{\theta}$ ) and the additional condition that $a_{i} \in V\left(T_{i}\right)$. Since $T_{\theta} \in \mathcal{T}$ and $T_{\theta}$ is path-maximal, it follows 
that each $T_{i}$ contains a maximal path $P_{i}=v z_{1}^{(i)} \ldots z_{j_{i}}^{(i)}$ of length at most $j$ such that $a_{i}=z_{1}^{(i)}$, and $z_{j_{i}}^{(i)}$ has a free color; and, more generally, there is a color $c^{(i)} \in L\left(z_{j_{i}-1}^{(i)}\right) \backslash\left\{\theta\left(z_{j_{i}-1}^{(i)}\right)\right\}$, such that any vertex at level $j_{i}$ in $H_{i}^{\prime}$ that is adjacent to $z_{j_{i}-1}^{(i)}$ and colored $c^{(i)}$ has a free color. We call such a path in $T_{i}$ good.

We shall now prove that there is an $L$-coloring $\varphi$ of $H-v$ such that $v$ is not adjacent to any vertex colored $c_{1}$, and thus there is an $L$-coloring of $H$.

For $i \in\{1, \ldots, r\}$, a colorful tree $J$ with root $a_{i}$ is a tree in $H_{i}^{\prime}-v$ such that:

- $a_{i} \in V(J)$ and all vertices of $J$ lie on $(\theta, L)$-alternating paths with origin at $v$, and

- if a vertex $u \in V(J)$ at level $q, 1 \leq q \leq j-1$ has a neighbor in $J$ at level $q+1$ colored $c$ under $\theta$, then $J$ contains all neighbors of $u$ at level $q+1$ with color $c$ under $\theta$; and, moreover, all neighbors of $u$ in $J$ at level $q+1$ is colored $c$ under $\theta$.

A colorful tree $J$ with root $a_{i}$ in $H^{\prime}$ is correct if for each maximal path $P^{\prime}$ in $J$ with origin at $a_{i}$, the terminus of $P^{\prime}$ has a free color.

Claim 4.4. For $i=1, \ldots, r$, the graph $H_{i}^{\prime}$ contains a correct colorful tree.

This claim can easily be proved by using the fact that each $T_{i}$ contains a good path. Since suppose that there is no correct colorful tree in $H_{i}^{\prime}$ for some $i \in\{1, \ldots, r\}$. Then every colorful tree in $H_{i}^{\prime}$ is not correct, which contradicts that $T_{i}$ satisfies (a), (c), (d), is path-maximal, and has a good path.

By the claim above, for $i=1, \ldots, r, H_{i}^{\prime}$ contain a correct colorful tree $J_{i}$. By recoloring all leaves of each $J_{i}$ with its free color, recoloring all other vertices of $J_{i}$ with the (unique) color of its children, and retaining the color of every other vertex of $H-v$, we obtain an $L$-coloring $\varphi$ of $H-v$ such that $v$ is not adjacent to any vertex colored $c_{1}$, implying the required contradiction.

Remark 4.5. If $T$ is a rooted $k$-proper tree with root $v$ in a graph $G$, and $L$ is a list assignment for $G$ such that $T$ is tree-bad, and we define $R(x)$ to be distance between $v$ and $x$ for any vertex $x$ of $T$, then $(T, v, R)$ is an $L$-bad proper triple according to the definition in Section 2. So for graphs with girth $g>3$, Lemma 4.3 provides a stronger characterization of which list assignments do not contain a proper coloring of the graph compared to Lemma 2.1.

Let us now prove Proposition 4.2 .

Proof of Proposition 4.2. Let $G=G(n)$ be a graph on $n$ vertices with maximum degree $\Delta=\Delta(n)$ and girth at least $g$, where $g$ is a fixed positive integer. Suppose further that $k$ is a fixed integer satisfying $k \geq 3$ and that $L$ is a random $(k, \mathcal{C})$-list assignment for $G$. We need to prove that if $\sigma=\omega\left(n^{\frac{1}{Q(k)-1}} \Delta\right)$, where $Q(k)$ is given by (7), then whp $G$ is $L$-colorable. By Lemma 4.3, it suffices to prove that whp $G$ does not contain a tree-bad odd (even) rooted $k$-proper tree if $g$ is odd (even).

We will prove the proposition in the case when $g$ is odd; the case when $g$ is even is similar.

As pointed out above, a rooted $k$-proper tree has $Q(k)$ vertices, where $Q(k)$ is given by (7). Hence, the number of odd rooted $k$-proper trees in $G$ is at most $n \Delta^{Q(k)-1}$. Given such a tree $T$ with root $v$, the number of ways of choosing the list assignment $L$ for $T$ such that $T$ is $(L$, tree)-bad is at most

$$
\sigma^{Q(k)-1}\left(\begin{array}{l}
\sigma-1 \\
k-1
\end{array}\right)^{k(k-1)^{\frac{g-3}{2}}}
$$


because there are less than $\sigma^{Q(k)-1}$ ways of choosing a proper coloring $\varphi$ of $T-v$, and there are thereafter at most $\left(\begin{array}{c}\sigma-1 \\ k-1\end{array}\right)^{k(k-1)^{\frac{g-3}{2}}}$ ways of choosing the remaining colors of the lists for the vertices at distance $\frac{g-1}{2}$ from $v$. Note that by conditions (i) and (ii) above, the list of every vertex at distance at most $\frac{g-3}{2}$ from $v$ in $T$ is determined, as soon as we have chosen the coloring $\varphi$ of $T-v$.

Denote by $X$ a random variable counting the number of tree-bad odd rooted $k$-proper trees in $G$. By the preceding paragraphs we have

$$
\begin{aligned}
\mathbb{E}[X] & \leq \frac{n \Delta^{Q(k)-1} \sigma^{Q(k)-1}\left(\begin{array}{c}
\sigma-1 \\
k-1
\end{array}\right)^{k(k-1)^{\frac{g-3}{2}}}}{\left(\begin{array}{c}
\sigma \\
k
\end{array}\right)^{Q(k)}} \\
& \leq A \frac{n \Delta^{Q(k)-1} k^{2 Q(k)}}{\sigma^{Q(k)-1}} \\
& =o(1),
\end{aligned}
$$

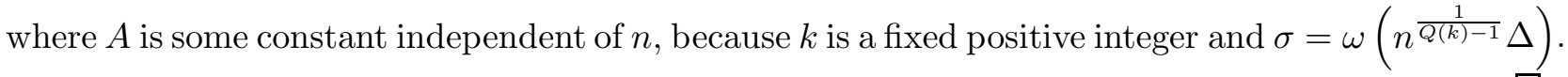

Finally, we have the following result for graphs with large girth.

Proposition 4.6. Let $G=G(n)$ be a graph on $n$ vertices with maximum degree at most $\Delta$ (where $\Delta$ is either constant or an increasing function of $n$ ) and girth $g=g(n)$ and let $L$ be a random $(k, \mathcal{C})$-list assignment for $G$. Then

(i) if $k=2, g \geq C \log n$, where $C>0$ is a fixed constant, and $\sigma(n) \geq A \Delta \log n$, where $A=A(C)$ is a fixed constant satisfying $A>4 e^{1 / C}$, then whp $G$ is L-colorable;

(ii) for each constant $C_{2}>0$, there are constants $k_{0}=\left\lceil\exp \left(2 / C_{2}\right)+1\right\rceil$ and $B_{0}=\exp \left(\frac{k_{0}-2}{2}\right) k_{0}^{2}$ such that if $g \geq C_{2} \log \log n, k \geq k_{0}, B>B_{0}$ and $\sigma(n) \geq B \Delta$, then whp $G$ is L-colorable.

Proof (sketch). We first prove part (i). We shall prove that whp $G$ has no $L$-coloring $\varphi$ such that it contains a $(\varphi, L)$-alternating path of length at least $g$. Since an $L$-critical induced subgraph of $G$ has minimum degree 2, by Lemma 2.1, this means that $G$ has no $L$-critical induced subgraph, which implies the desired result.

As in the proof of Theorem 1.4 the expected number of paths $P$ in $G$ on at least $g$ vertices, for which there is an $L$-coloring $\varphi$ of $P-v$, where $v$ is the origin of $P$, such that $P$ is $(\varphi, L)$-alternating is at most

$$
\sum_{r=g}^{n} \frac{n \Delta^{r-1} k^{2 r}}{\sigma^{r-1}}=o(1),
$$

provided that $k=2, g \geq C \log n$ and $\sigma(n) \geq A \Delta \log n$, where $A>4 e^{1 / C}$.

Let us now prove part (ii). Evidently, it suffices to prove the theorem in the case when $k=k_{0}$. The proof is almost identical to the proof Proposition 4.2. Arguing as in that proof it suffices to prove that the expression

$$
\begin{aligned}
& \frac{n \Delta^{Q(k)-1} \sigma^{Q(k)-1}\left(\begin{array}{c}
\sigma-1 \\
k-1
\end{array}\right)^{k(k-1)^{\frac{g-3}{2}}}}{\left(\begin{array}{c}
\sigma \\
k
\end{array}\right)^{Q(k)}} \\
& \leq \frac{n \Delta^{Q(k)-1} k^{2 Q(k)}}{\sigma^{Q(k)-1}}
\end{aligned}
$$


tends to 0 as $n \rightarrow \infty$. Noting that for large $g, Q(k) \geq \frac{2}{k-2}\left((k-1)^{g / 2}-1\right)$, it is easily verified that (9) tends to 0 as $n \rightarrow \infty$ provided that $k \geq \exp \left(2 / C_{2}\right)+1, \sigma(n) \geq B \Delta$ and $g \geq C_{2} \log \log n$, where $B>B_{0}=\exp \left(\frac{k_{0}-2}{2}\right) k_{0}^{2}$.

\section{Random lists of non-constant size}

In this section we demonstrate how the methods from Section 2 and 3 can be used for proving some analogous results on list coloring when the size $k$ of the lists in a random $(k, \mathcal{C})$-list assignment is a (slowly) increasing function of $n$. We shall derive such analogues of several of the results in the preceding sections.

There are some previous results on coloring graphs from random lists of non-constant size in the literature: In [2] it is proved that there is a constant $c>0$ such that if $L$ is a random $(k,\{1, \ldots, n\})$ list, assignment for $\mathcal{L}\left(K_{n, n}\right)$, where $\mathcal{L}\left(K_{n, n}\right)$ is the line graph of the balanced complete bipartite graph on $n+n$ vertices and $k>(1-c) n$, then whp there is an $L$-coloring of $\mathcal{L}\left(K_{n, n}\right)$. Note that in [2] this result is formulated in the language of arrays and Latin squares.

In [7] it is proved that for the complete graph $K_{n}$ on $n$ vertices the property of being colorable from a random from a random $(k,\{1, \ldots, n\})$-list assignment has a sharp threshold at at $k=\log n$. Moreover, a similar result for the line graph of the complete bipartite graph $K_{m, n}$ with parts of size $m$ and $n$, where $m=o(\sqrt{n})$ is also proved.

Let us now prove an analogue of Theorem 1.4. The proofs of all of the results in this section follow proofs in Section 2-4 quite closely, so in general, we omit proofs or just provide brief sketches. Througout this section we assume that $G=G(n)$ is a graph on $n$ vertices with maximum degree at most $\Delta$, and $L$ a random $(k, \mathcal{C})$-list assignment for $G$, where $k=k(n)$ and $\Delta=\Delta(n)$ are non-constant increasing integer-valued functions of $n$ satisfying $k<\Delta$.

Theorem 5.1. Suppose that $k=O\left(\log ^{1 / 4} n\right)$ and $\Delta=O\left(n^{1 / 3 k^{3}}\right)$. For any $\epsilon>0$ the following holds:

(i) If $k=o(\log \Delta)$ and $\sigma(n) \geq(1+\epsilon) n^{1 / k^{2}} \Delta^{1 / k} k$, then whp $G$ is L-colorable;

(ii) if $k=C \log \Delta$ and $\sigma(n) \geq(1+\epsilon) n^{1 / k^{2}} \exp (1 / C) k$, then whp $G$ is L-colorable;

(iii) if $k=\omega(\log \Delta)$ and $\sigma(n) \geq(1+\epsilon) n^{1 / k^{2}} k$, then whp $G$ is L-colorable.

The proof of the above theorem is almost identical to the proof of Theorem 1.4. Since $k$ is relatively small compared to $\sigma$ and $n$, the same arguments and calculations as in the proof of Theorem 1.4 yield the required conclusions, given that $k, \Delta$ and $\sigma$ satisfy the above conditions. The details are omitted.

Note further that the example after Thereom [1.4. shows that the bound on $\sigma$ in the above theorem is best possible up to the multiplicative factor $k$, provided that $k=o\left(\Delta^{1 / 2}\right)$.

Next, we have the following analogue of Proposition 2.5 for random lists of non-constant size.

Proposition 5.2. Let $\alpha$ and $s$ be constants satisfying $1<\alpha \leq 3$ and $s \geq 2+\gamma$, for some $\gamma>0$. For any $\epsilon>0$, if

- $k=O\left(\log ^{1 / \alpha-\delta} n\right)$ for some $\delta>0$,

- $\Delta=O\left(n^{1 / k^{\alpha}}\right)$, and 
- $\sigma(n) \geq(1+\epsilon) n^{1 / k^{\frac{\alpha+1}{2}}} \Delta^{s} k$

then $G$ is L-colorable.

Again, the proof is virtually identical to the proof of Proposition 2.5 and is therefore omitted.

For lists of greater size we have the following which is valid for all $\Delta$ :

\section{Proposition 5.3.}

(i) If $k=o(\log n)$, then for any $\epsilon>0$, if $\sigma(n) \geq(1+\epsilon) n^{1 / k} \Delta k$, then $\mathbf{w h p} G$ is L-colorable.

(ii) If $k \geq C \log n$, where $C$ is some constant, then for any $\epsilon>0$, if $\sigma \geq(1+\epsilon) C \exp (1 / C) \Delta \log n$, then whp $G$ is L-colorable.

Proof (sketch). The proofs of both part (i) and (ii) are similar to the proof of Proposition 2.6. Arguing as in that proof, it suffices to verify that the expression

$$
n\left(\begin{array}{l}
\Delta \\
k
\end{array}\right) \frac{\left(\begin{array}{l}
\sigma \\
k
\end{array}\right) k !\left(\begin{array}{c}
\sigma-1 \\
k-1
\end{array}\right)^{k}}{\left(\begin{array}{l}
\sigma \\
k
\end{array}\right)^{k+1}}=O\left(\frac{n \Delta^{k} k^{k}}{\sigma^{k}}\right)
$$

tends to 0 as $n \rightarrow \infty$.

Finally, we remark that it is possible to derive corresponding results for lists of non-constant size of Propositions 4.1 and 4.2 by proceeding as in the proofs of these propositions. Proposition 4.1 is valid for non-constant $k$ under the additional assumption that

$$
k=O\left(\log ^{\frac{1}{g}-\delta} n\right), \text { for arbitrarily small } \delta>0,
$$

and provided that $\sigma$ satisfies

$$
\sigma(n) \geq(1+\epsilon) n^{\frac{1}{(k-1) Q(k+1)+1}} \Delta^{1+\gamma} k, \text { for arbitrarily small } \gamma>0 \text { and } \epsilon>0 .
$$

Similarly, Proposition 4.2 is valid for non-constant $k$ provided that

- $k=o\left(\log ^{\left\lceil\frac{2}{g-2}\right\rceil} n\right)$ and $\sigma(n) \geq(1+\epsilon) n^{\frac{1}{Q(k)-1}} \Delta k^{2}$, or

- $k \geq C \log ^{\left\lceil\frac{2}{g-2}\right\rceil} n$, where $C$ is some constant, and $\sigma(n) \geq A \Delta \log ^{\left\lceil\frac{4}{g-2}\right\rceil} n$, where $A=A(C)$ is a suitably chosen constant.

\section{References}

[1] N. Alon, J. H. Spencer, The Probabilistic Method, 2nd ed, Wiley, New York, 2000.

[2] Lina J. Andrén, C. J. Casselgren, L.-D. Öhman, Avoiding arrays of odd order by Latin squares, Combinatorics, Probability Computing 22 (2013), 184-212.

[3] B. Bollobás, Modern Graph Theory, Springer, New York, 1998.

[4] C. J. Casselgren, Vertex coloring complete multipartite graphs from random lists of size 2, Discrete Mathematics 311 (2011), pp. 1150-1157. 
[5] C. J. Casselgren, Coloring graphs from random lists of size 2, European Journal of Combinatorics 33 (2012), 168-181.

[6] C. J. Casselgren, Coloring graphs from random lists of fixed size, Random Structures and Algorithms 44 (2014), 317-327.

[7] C. J. Casselgren, R. Häggkvist, Coloring complete and complete bipartite graphs from random lists, Graphs and Combinatorics (in press).

[8] P. Erdős, A. L. Rubin, H. Taylor, Choosability in graphs, Proceedings West Coast Conf. on Combinatorics, Graph Theory and Computing, Congressus Numerantium XXVI, 1979, pp. $125-157$.

[9] M. Krivelevich, A. Nachmias, Coloring powers of cycles from random lists, European Journal of Combinatorics 25 (2004), pp. 961-968.

[10] M. Krivelevich, A. Nachmias, Coloring Complete Bipartite Graphs from Random Lists, Random Structures and Algorithms 29 (2006), pp. 436-449.

[11] V. G. Vizing, Coloring the vertices of a graph with prescribed colors, Metody Diskretnogo Analiza Teorii Kodov i Skhem 29 (1976), pp. 3-10 (in Russian). 